\section{Case Reports in Oncology}

\title{
Duodenal Metastasis of Pulmonary Pleomorphic Carcinoma: A Case Report
}

\author{
Masayuki Matsuda ${ }^{a}$ Yoshiro Kai ${ }^{a}$ Suzuka Harada ${ }^{b}$ Kentaro Suzukia \\ Shigeto Hontsu ${ }^{c}$ Shigeo Muro ${ }^{c}$ \\ aDepartment of Respiratory Medicine, Minami-Nara General Medical Center, Nara, Japan; \\ ${ }^{b}$ Department of Surgery, Minami-Nara General Medical Center, Nara, Japan; 'Department of \\ Respiratory Medicine, Nara Medical University, Nara, Japan
}

\section{Keywords}

Pleomorphic carcinoma $\cdot$ Duodenal metastasis $\cdot$ Pembrolizumab

\begin{abstract}
Pulmonary pleomorphic carcinoma (PPC) is a rare subtype of lung sarcomatoid carcinoma that has a poor prognosis, and no standard therapy has been established. Here, we report the case of a 74-year-old man with PCC who showed rare duodenal metastasis. He was referred to our hospital with a mass shadow in the right lung. The patient was diagnosed with PPC clinical stage II B on the basis of immunohistochemical staining from bronchoscopy, and the tumor proportion score of programmed death-ligand 1 was $80 \%$. He did not want any treatment. A year and a half later, progressive anemia was detected. The primary tumor was stable; however, abdominal computed tomography and esophagogastroduodenoscopy revealed a duodenal tumor with stenosis. He was diagnosed with duodenal metastasis from PPC, and he underwent gastrojejunal bypass surgery to prevent bowel obstruction. After surgery, he received pembrolizumab for chemotherapy. However, owing to the progression of peritoneum dissemination, he died 2 months later due to the onset of melena.
\end{abstract}

\section{Introduction}

Pulmonary pleomorphic carcinoma (PPC) is defined as a group of poorly differentiated nonsmall cell carcinomas and comprises $0.1 \%-0.4 \%$ of all lung cancers [1]. PPC has a progressive clinical course and a worse outcome. Duodenal metastasis is also a rare metastasis of nonsmall 


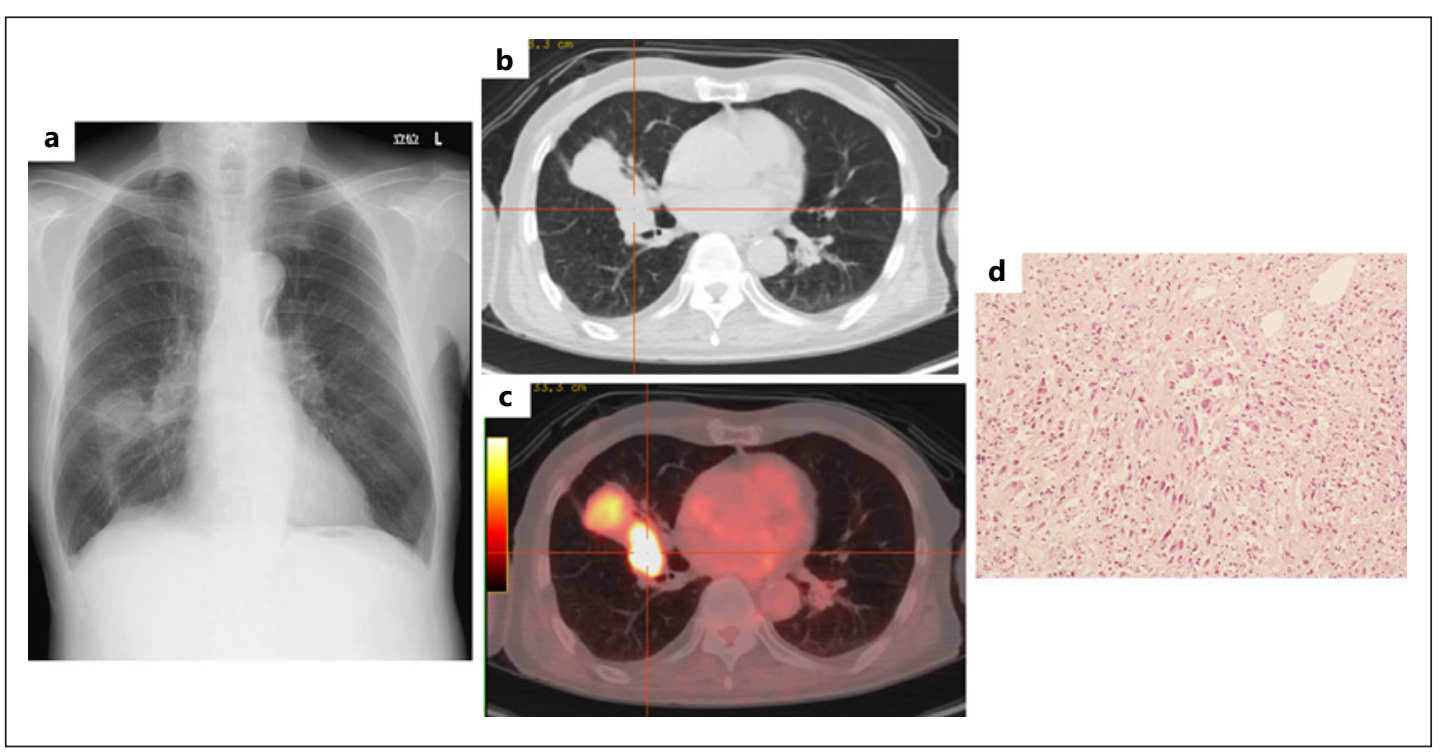

Fig. 1. a Chest X-ray shows a mass in the right lower field. b CT scan of the chest shows a tumor shadow and atelectasis in the right middle lobe. c F18-fluorodeoxyglucose position emission tomography scan showed high uptake in the right lung. $\mathbf{d}$ Hematoxylin and eosin stain. $\times 100$. Microscopic analysis of the lung biopsy revealed the growth of poorly differentiated malignant cells, including spindle cells and giant cells.

cell carcinoma. PPC has a poor prognosis. Recently, immune therapy has been reported to be effective in treating PPC [2]. However, standard therapy has not been established. Metastasis from PPC to other organs, including the brain, liver, adrenal tissue, and bone, has been reported. We report a case of a patient with duodenal metastasis from PPC.

\section{Case Report/Case Presentation}

A 74-year-old man, who had undergone treatment for hypertension and was on oral medication, complained of wet cough and was referred to our hospital for a mass in the right lung. He had a history of 27 pack-years of smoking. Breath sounds were slightly diminished in the right lung. Blood counts and biochemistry findings were within normal ranges. Carcinoembryonic antigen, cytokeratin 19 fragments, and pro-gastrin-releasing peptide were within normal ranges. Chest X-ray revealed a lesion in the hilar region and lower lung area of the right lung (Fig. 1a). Chest CT revealed the presence of a mass and atelectasis in the right middle lobe (Fig. 1b). F18-fluorodeoxyglucose positron emission tomography was performed, revealing high uptake in the hilar lesion (Fig. 1c). Histological examination of transbronchial biopsy specimens revealed that the tumor was composed primarily of giant cells and spindle cells (Fig. 1d). The patient was diagnosed with PCC, cT2aN1M0 stage II B. Operation was recommended, but he chose not to receive treatment. Lung function tests showed obstructive impairment (forced expiratory volume in $1 \mathrm{~s}$ [FEV1], 1.31 L; predicted FEV1 [\%FEV1], 49.3\%; FEV1/forced vital capacity [FVC], 40.5\%), indicating chronic obstructive pulmonary disease; thus, treatment was initiated only for chronic obstructive pulmonary disease.

A year and a half later, he developed general fatigue and shortness of breath. Laboratory data showed severe anemia (hemoglobin $6.2 \mathrm{~g} / \mathrm{dL}$ ). He tested positive for melena on digital examination. On chest X-ray, the size of the primary mass in the right field was stable, and atelectasis was not present (Fig. 2a). Abdominal enhanced CT showed a mass in the second 


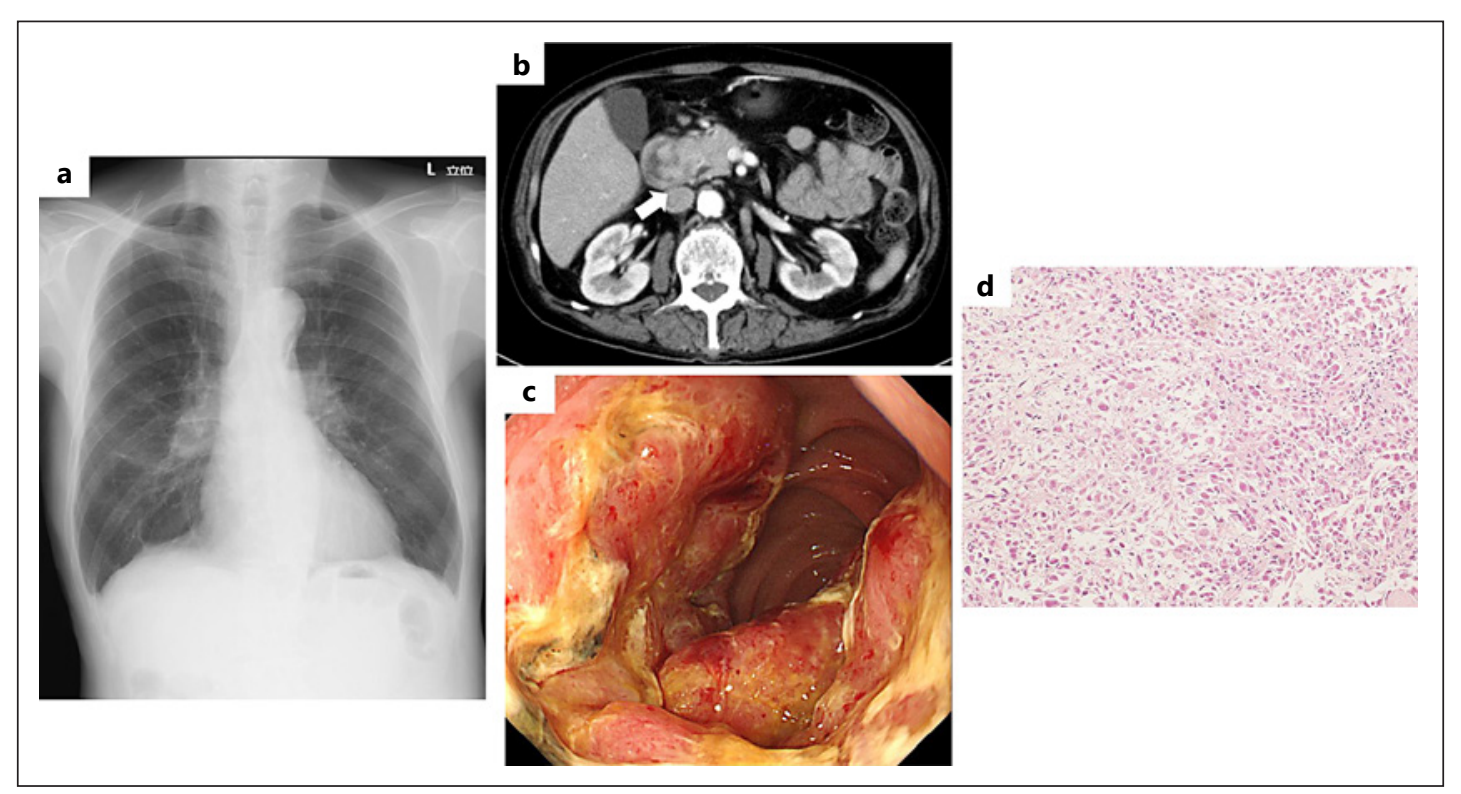

Fig. 2. a Chest X-ray taken a year and a half later. The size of the primary mass in the right field was stable, and atelectasis was not present. b Abdominal CT a year and a half later shows a mass in the duodenum. c Esophagogastroduodenoscopy demonstrated a bleeding lesion with an ulcer and stenosis in the second (descending) part of the duodenum. d Hematoxylin and eosin stain. $\times 100$. The histologic appearance of the duodenal tumor is similar to that of the lung tumor.

part of the duodenum (Fig. 2b). Esophagogastroduodenoscopy showed 3/4 circumferential type 3 tumor with an ulcer and stenosis (Fig. 2c). Biopsy of the duodenal mass showed PCC (Fig. 2d). This duodenal lesion was immunohistochemically consistent with the lung lesion, showing a tumor proportion score of $80 \%$ for programmed death-ligand 1 . He opted to treat it. Gastrojejunal bypass was performed to prevent bowel obstruction, followed by systemic chemotherapy (pembrolizumab). However, owing to aggressive intraperitoneal progression, he died 2 months later due to the onset of melena.

\section{Discussion/Conclusion}

PCC is a rare and aggressive subtype of lung cancer. Its incidence is reported to be $0.1 \%$ $0.4 \%$ of all lung cancers [1]. It is related to smoking history, and it has more aggressive growth and a poorer outcome compared with other types of nonsmall cell carcinomas. PCC prognosis is poor, with patients reportedly having 8-19 months to live once diagnosed [1]. Furthermore, gastrointestinal metastasis of lung cancer is rare; its incidence is reported to be $0.2 \%-1.7 \%$ of all lung cancers [3]. Lung cancer with gastrointestinal metastasis has worse prognosis than lung cancer without gastrointestinal metastasis. The mean time from lung cancer diagnosis to identification of gastrointestinal metastasis is reported to be 13.5 months, and the mean time from identification of gastrointestinal metastasis to death is 100.6 days [3]. In our case, a year and a half later, the primary tumor was stable; however, PCC metastasized to the duodenum, presenting with anemia and upper gastrointestinal bleeding. PCC is known to be resistant to chemotherapy and radiotherapy, and no standard therapy has been established. Recently, it has been reported that sarcomatoid carcinoma is more likely to exhibit high levels of PD-L1 expression compared with nonsmall cell lung cancer. In previous studies, 53.0\%$90.2 \%$ of sarcomatoid carcinomas showed positive PD-L1 expression [4], and immunotherapy 
was effective in treating sarcomatoid carcinoma [5]. In this case, after duodenal bypass, we introduced pembrolizumab because of the high PD-L1 expression. However, it was not effective; performance status worsened, and he died 2 months later due to the onset of melena. To the best of our knowledge, only 2 cases of small bowel metastasis from PPC have been reported [6,7]. Furthermore, there is only 1 report of duodenal metastasis of PCC, which is a very rare case [8]. In conclusion, PPC that metastasized to the duodenum is very rare. In the case of atypical duodenal tumor, metastatic duodenal tumor must be considered.

\section{Acknowledgment}

The authors would like to thank Enago (www.enago.jp) for the English language review.

\section{Statement of Ethics}

The study is exempt from ethics committee approval. Written informed consent was obtained from the patient and his family for publication of this case report and any accompanying images.

\section{Conflict of Interest Statement}

The authors have no conflicts of interest to declare.

\section{Funding Sources}

The authors did not receive any funding.

\section{Author Contributions}

M.M. and Y.K. wrote the manuscript. All the authors have participated in this case.

\section{Data Availability Statement}

All data generated or analyzed during this study are included in this article. Further enquiries can be directed to the corresponding author.

\section{References}

1 Chang YL, Lee YC, Shih JY, Wu CT. Pulmonary pleomorphic (spindle) cell carcinoma: peculiar clinicopathologic manifestations different from ordinary non-small cell carcinoma. Lung Cancer. 2001 Oct;34(1):91-7.

2 Fujimoto E, Yokoi T, Mikami K, Kanemura S, Shibata E, Horio D, et al. Successful treatment of pulmonary Pleomorphic Carcinoma with Nivolumab: a case report. Case Rep Oncol. 2018 May-Aug;11(2):336-40.

3 Taira N, Kawabata T, Gabe A, Furugen T, Ichi T, Kushi K, et al. Analysis of gastrointestinal metastasis of primary lung cancer: clinical characteristics and prognosis. Oncol Lett. 2017 Aug;14(2):2399-404.

4 Yvorel V, Patoir A, Casteillo F, Tissot C, Fournel P, Stachowicz ML, et al. PD-L1 expression in pleomorphic, spindle cell and giant cell carcinoma of the lung is related to TTF-1, p40 expression and might indicate a worse prognosis. PLoS One. 2017;12(7):e0180346. 
5 Kotlowska MP, Rueda AG, Olmedo ME, Benito A, Roldán AS, Fernandez Méndez MA, et al. Efficacy of immunotherapy in sarcomatoid lung cancer, a case report and literature review. Respir Med Case Rep. 2019;26:310-4.

6 Hara S, Hosoi K, Suga Y, Takata T, Degami H, Kinoshita Y, et al. Multiple cytokines-producing pleomorphic carcinoma of lung with metastasis to the small intestine. Ann Thorac Cardiovasc Surg. 2014;20 Suppl:666-8.

7 Koh H, Chiyotani A, Tokuda T, Suzumura H, Kamiishi N, Takahashi H, et al. Pleomorphic carcinoma showing rapid growth, multiple metastases, and intestinal perforation. Ann Thorac Cardiovasc Surg. 2014;20 Suppl: 669-73.

8 Ustaoğlu M, Koçak G, Siviloğlu Ç, Cengiz E. An unusual case of duodenal metastasis of pulmonary pleomorphic carcinoma. Turk J Gastroenterol. 2018 Jan;29(1):136-7. 